\title{
BMJ Open The 20 most important questions for novices of full-endoscopic spinal surgery in China: a mixed-method study protocol
}

\author{
Can Liu, ${ }^{1}$ Hong Ling Chu (D) , ${ }^{2}$ Geng Li, ${ }^{3}$ Jiao Yang He, ${ }^{3}$ Yun Long Ma, ${ }^{3}$ \\ Chuan Chao Du, ${ }^{4}$ Bao Hai (D) , ${ }^{4}$ Bin Zhu ${ }^{5}$
}

To cite: Liu C, Chu HL, Li G, et al. The 20 most important questions for novices of fullendoscopic spinal surgery in China: a mixed-method study protocol. BMJ Open 2021;11:e049902. doi:10.1136/ bmjopen-2021-049902

- Prepublication history for this paper is available online To view these files, please visit the journal online (http://dx.doi. org/10.1136/bmjopen-2021049902).

$\mathrm{CL}$ and $\mathrm{HLC}$ are joint first authors.

Received 07 February 2021 Accepted 09 August 2021

\section{ABSTRACT}

Introduction The full-endoscopic spinal surgery (FESS) procedure is widely accepted and welcomed in China. With the continuous development of minimally invasive surgery, the further expansion of indications and the greater diversity of techniques, spinal endoscopic surgery currently accounts for more than $10 \%$ of spinal surgery in China, ranking among the top in the world. However, the admission system and standardised training system for spinal endoscopic surgery are not perfect, which presents a challenge and disadvantage for novices.

Methods and analysis Exploratory mixed methods are applied for designing this study. First, we will collect questions from novices by allowing them to openly list their concerns to those who have completed FESS. These qualitative questions will be categorised using NVivo software. To produce the qualitative results, a questionnaire for the sequential two-round Delphi approach will be developed to identify the 20 most important questions from novices. This study is planned to be started at April 2021, and completed at March 2022.

Ethics and dissemination The Research Ethics Committee of Peking University Third Hospital provided a waiver for this Delphi protocol. We expect that the findings will be published in a clinical journal and presented at conferences. Furthermore, we hope that the results can contribute to answering the questions raised by novices of spinal endoscopy in the form of books and to improving the training system for spinal endoscopy surgery.

\section{INTRODUCTION}

Since the introduction of percutaneous endoscopic lumbar discectomy (the 'YESS' technique) to China in the late 1990 s, endoscopic spinal surgery has developed rapidly in recent decades with increased acceptance. ${ }^{1}$ Especially in recent years, full-endoscopic spinal surgery (FESS) has become the main treatment for degenerative diseases of the spine in some economically developed areas. The indications for surgery have expanded from simple lumbar disc herniation to spinal degenerative diseases, infections and even tumours; indications include extreme lateral
Strengths and limitations of this study

- Exploratory mixed methods design is used to collect questions openly from novices of full-endoscopic spinal surgery (FESS) by using qualitative approach, and sequentially conduct Delphi survey to screen the most important questions mainly by using quantitative approach, which might be a good example for researchers to solve the similar research questions.

- All questions are provided by novices of FESS who are in the learning curve stage in China, which are real-world problems.

- The consultation experts consist of doctors with a large number of annual FESSs in China who have rich surgical experience and a high reputation.

- 'The most important question' about FESS means that the evidence-based answer to this question is crucial for novices of FESS to successfully complete the learning curve and avoid risks in the actual operation.

- To the best of our knowledge, this is a rare study that generates Delphi-based expert consensus on the promotion and application of a new technology.

disc herniation, recurrent disc herniation, migrated disc herniation, lumbar infectious spondylitis, discogenic low back pain and spinal tumours. ${ }^{2-7}$ With the expansion of indications, the number of endoscopic spinal surgeries has also increased. It is estimated that 50000 to 60000 spinal endoscopic surgeries are performed in China each year, accounting for approximately $10 \%$ of spinal surgeries, and this proportion is growing rapidly every year. ${ }^{1}$

In recent decades, with the development of techniques from YESS $^{8}$ and transforaminal endoscopic spine system (TESSYS) ${ }^{9}$ to fullendoscopic techniques, ${ }^{10}$ endoscopic spinal surgery has been continuously innovated and improved, which has advanced the safety and efficacy of surgery. It has become the 
foundation for the continual extension of indications for endoscopic spinal surgery.

Many clinical departments in China perform FESS, especially full-endoscopic lumbar discectomy operations, including orthopaedics, pain centres, neurosurgery, rehabilitation, interventional medicine and sports medicine. ${ }^{1}$ As a clinical technique, its learning curve typically includes theoretical learning and clinical practice, and there are many ways to evaluate surgical outcomes. However, there is no firm consensus on strategies to improve the learning curve regarding the characteristics of the FESS technique. ${ }^{112}$ The lack of a training system has become a bottleneck for novices of FESS (ie, within their first 20 cases) to perform surgery. ${ }^{13} 14$ Their concerns regarding FESS mainly include a steep learning curve, fear of failure, complications and recurrence after surgery. ${ }^{15}$ The complications involves in novices' implementation of this technology indicate the need to quickly establish a standardised training system and access system. ${ }^{16}{ }^{17}$ It is believed that the learning curve, though steep, can be overcome through strict standard training and suitable patient selection. ${ }^{18}$ In the establishment of relevant systems and training systems, mature surgeons' experience and technical understanding of spinal endoscopic surgery are extremely valuable. ${ }^{19}$

The Delphi method is a collective anonymous consensus process in the form of correspondence consultation. It is characterised by its anonymity, multiple feedback and other statistics. Furthermore, it is an established method of drawing on the opinions of often diverse groups of experts on practice-related problems. ${ }^{20}{ }^{21}$ Therefore, we designed two rounds of Delphi expert email consultations to achieve the purpose of our project, making full use of the practical experience and theoretical understanding of different experts and avoiding the result bias caused by face-to-face meetings.

\section{OBJECTIVE}

The aim of this mixed-method study is to identify the 20 most important questions in the area of FESS among a large number of spinal endoscopic surgery doctors in China.

\section{METHODS AND DESIGN Study design}

An exploratory sequential mixed-methods design will be used in this study, with initial qualitative data collection and analysis informing subsequent quantitative data collection and analysis that integrates the strengths of inductive and deductive reasoning. In phase 1, we plan to explore questions from novices of FESS. In phase 2, we will conduct a two-round Delphi survey to identify the 20 most important questions. A summary of the research design is presented in figure 1 .

\section{Study setting and participants}

The working group consists of physicians with comparable experience in FESS, epidemiologists and graduate students who act as the designers and implementers of the programme. Agreement will be reached concerning participant selection, consensus thresholds, the survey format and question structure, and the analysis process according to the proposed quality indicators for a Delphi study. The consultation panellists comprise spinal endoscopic surgery doctors with rich surgical experience and a high reputation in China. We will recruit consultation experts following principles of authority and representativeness from all provinces in mainland China through the Minimally Invasive Spine Expert Committee.

\section{Selection criteria}

1. Engaged in spinal endoscopy.

2. More than 200 cases of endoscopic operations in 1 year.

3. More than 1000 total cases of spinal endoscopic surgery.

4. Support and willingness to participate in this research.

5. Complete multiple rounds of correspondence.

\section{Sample size}

Approximately 100 experts distributed at Peking University Third Hospital and other areas in mainland China will be invited to participate to complete both the qualitative and quantitative two-round e-Delphi surveys.

\section{DATA COLLECTION}

For this exploratory mixed-methods study, qualitative data will initially be collected from participants by requesting that they openly list their concerns to those who have completed FESS in phase 1. Sequentially, quantitative data will be collected by using a two-round Delphi survey in phase 2. The process of implementation is illustrated in figure 2.

\section{Phase I Phase II}

What are the questions that Importance of these questions

Refining 20 most important concern FESS doctors? from experts' perspective questions

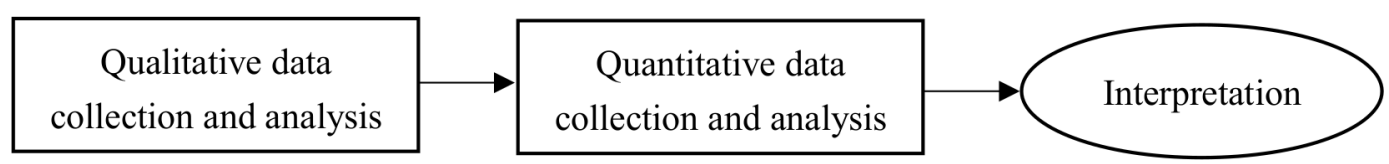

Figure 1 Explanatory sequential mixed-methods design of the study. FESS, full-endoscopic spinal surgery. 
Title: The 20 most important questions for novices of Full-endoscopic Spinal surgery

\section{Purpose}

Qualitative

\begin{tabular}{|l|}
\hline To explore \\
novices' questions \\
about FESS \\
\end{tabular}

\section{Quantitative}

\begin{tabular}{|l|}
\hline To identify the 20 \\
most important \\
questions in the \\
area of FESS \\
\end{tabular}
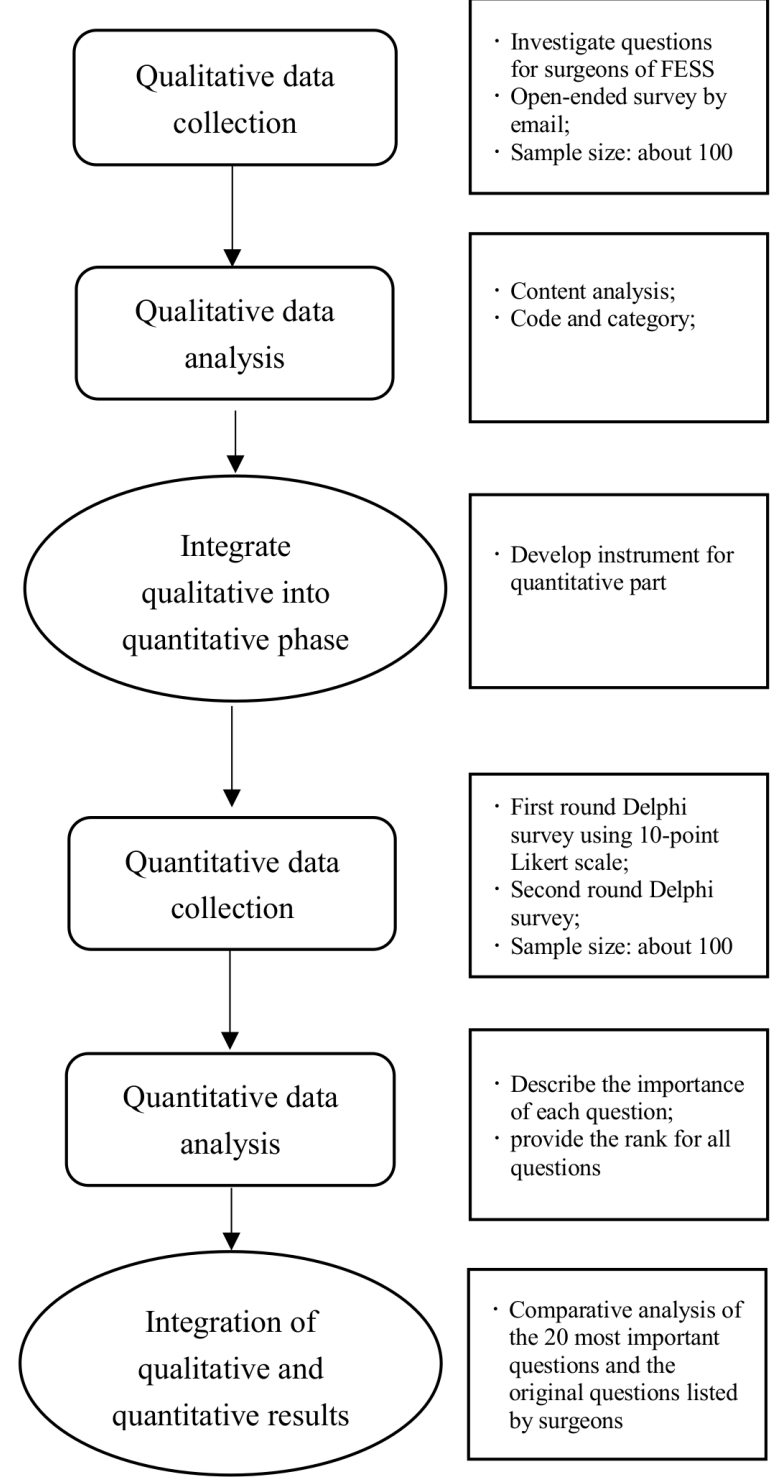

Sample size: about 100
Develop instrument for quantitative part

\section{Procedure}

Investigate questions for surgeons of FESS

Open-ended survey by

email;

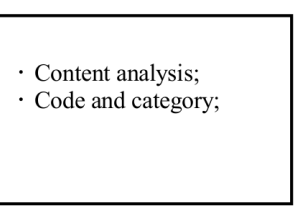

- Domains of questions;

- Questions after

category;
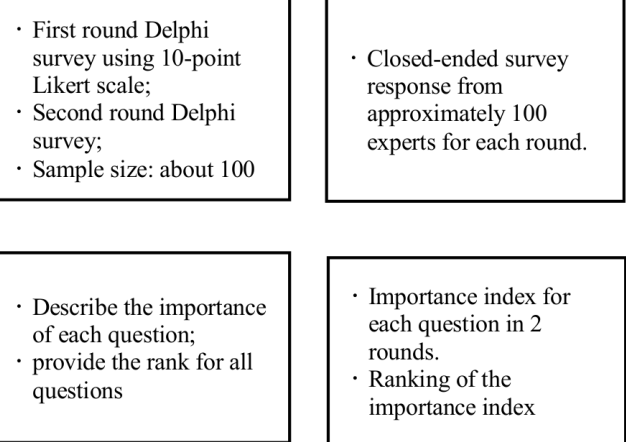

- Importance index for each question in 2 rounds.

- Ranking of the

importance index

$$
\begin{aligned}
& \text { Comparative analysis of } \\
& \text { the } 20 \text { most important } \\
& \text { questions and the } \\
& \text { original questions listed } \\
& \text { by surgeons }
\end{aligned}
$$

Figure 2 An overview of an exploratory sequential mixed-methods research design in the proposed research. Delphi data collection and analysis sequence. FESS, full-endoscopic spinal surgery.

\section{Phase I}

Collecting qualitative questions to form a problem database

Open questions about the most difficult questions, the questions that most require solutions, or the issues of greatest concern encountered during the operation for novices of FESS will be sent to approximately 100 doctors at the pain medicine centre of Peking University Third Hospital by email by the working group. The feedback will be refined and classified to develop a problem database with a total of $\mathrm{N}$ subquestions in $\mathrm{M}$ categories by NVivo qualitative data analysis software ${ }^{22}$ (NVivo 11 pro). The working group specialises in language expression and modification of each problem through discussion and codes each of them with N-M.

\section{Generating the instrument}

Based on the coded problem database, the instrument for the first round of Delphi will be sequentially developed. The invitation letter sends a warm invitation to the consultation panellists and briefly introduces the significance, scheme and matters for attention of this consultation project. The questionnaire consists of two parts. The first part includes the basic information and the professional discipline of the participant. The second 
part lists $\mathrm{N}$ questions divided into $\mathrm{M}$ categories. Each of the $\mathrm{N}$ question items is followed by a grid in which the panellists are asked to score the importance of these questions. The assessment of importance can be judged by a score from 0 (least important) to 9 (most important). Blank areas are left for participants to suggest other necessary question items. This process allows us to define the 'most important' questions: a higher score means that the evidence-based answer to this question is crucial for novices of FESS to successfully complete the learning curve and avoid risks in the actual operation.

\section{Phase II}

Two-round e-Delphi study

Delphi method rounds: round 1

In the first round, the consultation questionnaire will be sent to all experts by email. The experts are invited to complete the questionnaire inquiry according to the instructions and then return the questionnaire by email. A 1-month turnaround time will be given for panel members to complete the questionnaire.

All panellists are invited to provide professional information, job familiarity and so on. More importantly, the panellists rank the importance of the $\mathrm{N}$ questions. A 10 -point Likert scale is used, ranging from $0=$ not at all important to $9=$ extremely important. ${ }^{23}$ Space is included at the end of the questionnaire for participants to provide comments and suggestions.

\section{Round-2 instrument}

After completing the first round of expert email consultation, we will collect all results for statistical analysis and form the second-round correspondence questionnaire according to the feedback. We will modify the list of question items, including those regarded as the most important (9 points) by at least $10 \%$ of panellists in the first round. ${ }^{24} \mathrm{~A}$ summary table of the results, including group ratings and comments obtained from round 1 , will be prepared. This table is used to provide controlled feedback and statistical group responses to participants. Space will be provided at the end of each question for comments and suggestions.

\section{Delphi method rounds: round 2}

For round 2, each panellist will be invited to complete the second round of the correspondence questionnaire. The requirements and criteria are the same as in the first round. Only those participants who completed the first round will be invited for the second round. Panellists are also provided with the summary table from round 1 . In both rounds, we will send up to three reminders in cases of non-response.

\section{DATA ANALYSIS}

After the consultation questionnaire is completed, the corresponding database will be established, and the data will be entered for parallel consistency and verification. For questions or missing data, experts will be contacted to confirm or complete the responses. The main analysis items and methods of the two rounds of consultation forms are as follows, referring to the relevant calculation methods of the Delphi method. ${ }^{24-27}$

\section{The questionnaire recovery rate $(\mathrm{H})$}

We will evaluate the enthusiasm of experts by the questionnaire recovery rate $(\mathrm{H})$. The questionnaire recovery rate is equal to the ratio of the total number of questionnaires recovered and the scores of questionnaires issued multiplied by $100 \%$.

\section{The authoritative coefficient (Cr)}

The authoritative coefficient $(\mathrm{Cr})$ is used to evaluate the reliability of expert scoring. $\mathrm{Cr}$ is determined by two aspects: (1) experts' familiarity with spinal endoscopy expertise (Cs), which is divided into five levels: very familiar, relatively familiar, generally familiar, not very familiar, unfamiliar; (2) the main judgement basis (Ca) of experts for scoring the importance of selected items is divided into practical experience, theoretical analysis and reference to domestic and foreign literature. Intuitively, judgement is the fourth aspect and is also divided into three levels of influence, and the corresponding coefficients are assigned differently. The sum of the four aspects of influence is $\mathrm{Ca}$. The calculation formula is $\mathrm{Cr}$ $=(\mathrm{Cs}+\mathrm{Ca}) / 2$.

\section{The full score rate $(K)$ and the total score $(S)$}

The importance of each item of the questionnaire is evaluated by the full score rate $(\mathrm{K})$ and the total score $(\mathrm{S})$ : K indicates the percentage of the full score obtained by this item as a percentage of the total items; $\mathrm{S}$ indicates the sum of the scores of this item.

\section{Coefficient of variation (CV) and Kendall's coefficient of concordance}

The coefficient of variation (CV) is used to evaluate the uniformity of expert opinions of various items, and Kendall's coefficient of concordance $(\omega)$ is used to evaluate the consistency of opinions of the entire questionnaire. The lower the $\mathrm{CV}$, the higher $\omega$, which indicates more unified opinions of experts and more convincing research results. The formula for the coefficient of variation $(\mathrm{CV})$ is $\mathrm{CV}=$ $\sigma /^{-} \mathrm{x}, \sigma$ is the SD of the indicator, and ${ }^{-} \mathrm{x}$ is the average of each indicator. Kendall's coefficient calculation method: the number of experts consulted with the questionnaire is $\mathrm{b}$, and the number of indicators to be evaluated is $\mathrm{k}$. Therefore this indicator reflects the degree of consistency of $b$ experts on the relative importance scores of all $k$ indicators. This indicator is represented by $\omega$ and is calculated by the following formula:

$$
\omega=\frac{12 \sum_{j=1}^{k} R_{j}^{2}-3 b^{2} k(k+1)^{2}}{b^{2} k\left(k^{2}-1\right)} .
$$

Note: $\mathrm{b}$ is the number of experts, $\mathrm{k}$ is the number of evaluation indicators, and $\mathrm{Rj}$ is the total rank of the $\mathrm{jth}$ observation object. 
The value of $\omega$ is between 0 and 1 ; the closer the value to 1 , the better the degree of agreement of all experts' evaluations of all indicators, which indicates better coordination among the experts in this round of research; otherwise, the opposite is true. SPSSV.17.0 can be used to calculate Kendall's coefficient $(\omega)$.

\section{Patient and public invovlement}

Patient and public will not be involved in the study.

\section{DISCUSSION}

Initial steps, tool development and dissemination are the three main stages of the framework for developing assessment tools. ${ }^{28}$ This study is situated in the dissemination stage by generating and seeking consensus on learning curves and technical points about spinal endoscopy. By integrating qualitative and Delphi expert consensus, we attempt to establish the most important issues so that novices can pay attention to the techniques of spinal endoscopy when performing it. Although this is the first study to provide a high-level solution to a technical problem for the promotion and application of a new technology, it provides directions and frameworks for us to organise experts and scholars to answer and interpret these questions professionally and accurately. We follow the evidence-based medicine model to seek precise solutions through rigorous design.

The development of spinal endoscopy has experienced a long process of improvement and practice, making this technique more widely used and more mature. Moreover, FESS is about to or has already become the mainstream in this area. It must be emphasised that the improvement and promotion of the technology and equipment are always derived from clinical practice, so the study of spinal endoscopy is inseparable from practice, and the experience and lessons learnt from practice are valuable and indispensable. ${ }^{14}$ This is also the reason why we chose experts with a large number of annual spinal endoscopic surgeries in China as the respondents. We have also designed indicators to evaluate the enthusiasm, professional familiarity and business ability of the experts to verify the authority and credibility of this study. Anonymity is always a valuable feature of Delphi research. ${ }^{29}$ We will complete the inquiry by e-mail (some experts use letters). The statistics and analysis of all data will be conducted by different personnel with the aim of maintaining objectivity and reducing deviation. We will present a consistent evaluation of expert opinions on the 20 selected questions and the entire questionnaire, not only to confirm the authority and authenticity of our results but also as a reminder that these questions are of great value in clinical application and technical learning. We will publish our research results in the form of a paper. In the next step, we will organise a group of experts engaged in spinal endoscopy surgery to discuss and answer these questions in depth. Then, a text version integrating feasibility, operability and practicability will be developed as answers to the questions and to overcome obstacles for novices of FESS.

We seek to identify the 20 most important questions with a rigorous design for novices of FESS. More importantly, our future answers to these questions and discussion results will provide a basis for the improvement of the training system and access system of spinal endoscopic surgery in the future as well as technology promotion and even technical improvement. Providing a feasible model for the promotion of other new technologies will be an additional benefit.

\section{ETHICS AND DISSEMINATION}

The Research Ethics Committee of Peking University Third Hospital provided a waiver for this Delphi protocol. This research is purely an effort towards the training and promotion of new technologies. All participation is voluntary, and we will ensure that respondents present their authentic views. We expect that the findings will be published in a clinical journal and presented at conferences. Furthermore, we hope to contribute to answering the questions of novices of spinal endoscopy in the form of books and to improve the training system for spinal endoscopy surgery.

\section{Author affiliations}

${ }^{1}$ Department of Orthopedics, Beijing First Hospital of Integrated Chinese and Western Medicine, Beijing, China

${ }^{2}$ The Clinical Epidemiology Research Center, Peking University Third Hospital, Beijing, People's Republic of China

${ }^{3}$ The Center for Pain Medicine, Peking University Third Hospital, Beijing, China ${ }^{4}$ Department of Orthopedics, Peking University Third Hospital, Beijing, China ${ }^{5}$ Department of Orthopedics, Capital Medical University Affiliated Beijing Friendship Hospital, Beijing, China

Contributors CL and HLC designed the study in collaboration, GL and JYH performed the data collection, YLM and CCD provided advice and guidance on the design. CL and BZ wrote the first draft of the protocol, HLC and BH provided final review of data and provided critical revision of draft and subsequent revision. All authors read and approved the final manuscript.

Funding This work was supported by the Capital's Funds for Health Improvement and Research (2020-2-4091); Key Clinical Projects of Peking University Third Hospital (BYSYZD2019001, BYSYZD2019017).

Competing interests None declared.

Patient and public involvement Patients and/or the public were not involved in the design, or conduct, or reporting, or dissemination plans of this research.

Patient consent for publication Not required.

Provenance and peer review Not commissioned; externally peer reviewed.

Open access This is an open access article distributed in accordance with the Creative Commons Attribution Non Commercial (CC BY-NC 4.0) license, which permits others to distribute, remix, adapt, build upon this work non-commercially, and license their derivative works on different terms, provided the original work is properly cited, appropriate credit is given, any changes made indicated, and the use is non-commercial. See: http://creativecommons.org/licenses/by-nc/4.0/.

\section{ORCID iDs}

Hong Ling Chu http://orcid.org/0000-0003-1846-9192

Bao Hai http://orcid.org/0000-0001-7404-918X

\section{REFERENCES}

1 Zhang X, Du J, Yeung AT. Development of Percutaneous Endoscopic Lumbar Discectomy (PELD)Technology in China. J Spine 2017;6:374-6. 
2 Ahn Y. Percutaneous endoscopic decompression for lumbar spinal stenosis. Expert Rev Med Devices 2014;11:605-16.

3 Yao $\mathrm{Y}$, Zhang $\mathrm{H}$, Wu J, et al. Comparison of three minimally invasive spine surgery methods for revision surgery for recurrent herniation after percutaneous endoscopic lumbar discectomy. World Neurosurg 2017;100:641-7.

4 Yeom K-seong, Choi Y-soo. Full endoscopic contralateral transforaminal discectomy for distally migrated lumbar disc herniation. J Orthop Sci 2011;16:263-9.

5 Wang X, Zhou S, Bian Z, et al. Unilateral percutaneous endoscopic debridement and drainage for lumbar infectious spondylitis. J Orthop Surg Res 2018;13:306.

6 Choi K-C, Shim H-K, Park CJ, et al. Usefulness of percutaneous endoscopic lumbar Foraminoplasty for lumbar disc herniation. World Neurosurg 2017;106:484-92.

7 Tsai S-H, Wu H-H, Cheng C-Y, et al. Full endoscopic Interlaminar approach for nerve root decompression of sacral metastatic tumor World Neurosurg 2018;112:57-63.

8 Yeung AT, Tsou PM. Posterolateral endoscopic excision for lumbar disc herniation: surgical technique, outcome, and complications in 307 consecutive cases. Spine 2002;27:722-31.

9 Hoogland T, Schubert M, Miklitz B, et al. Transforaminal posterolateral endoscopic discectomy with or without the combination of a low-dose chymopapain: a prospective randomized study in 280 consecutive cases. Spine 2006;31:E890-7.

10 Ruetten S, Komp M, Merk H, et al. Use of newly developed instruments and endoscopes: full-endoscopic resection of lumbar disc herniations via the interlaminar and lateral transforaminal approach. J Neurosurg Spine 2007;6:521-30.

11 Simonson DC, Roukis TS. Incidence of complications during the surgeon learning curve period for primary total ankle replacement: a systematic review. Clin Podiatr Med Surg 2015;32:473-82.

12 Ahn Y, Lee S, Son S, et al. Learning curve for Transforaminal percutaneous endoscopic lumbar discectomy: a systematic review. World Neurosurg 2020;143:471-9.

13 Wu X-B, Fan G-X, Gu X, et al. Learning curves of percutaneous endoscopic lumbar discectomy in transforaminal approach at the L4/5 and L5/S1 levels: a comparative study. J Zhejiang Univ Sci B 2016;17:553-60.

14 Wang $\mathrm{H}$, Huang $\mathrm{B}$, Li C, et al. Learning curve for percutaneous endoscopic lumbar discectomy depending on the surgeon's training level of minimally invasive spine surgery. Clin Neurol Neurosurg 2013;115:1987-91.
15 Son S, Ahn Y, Lee SG, et al. Learning curve of percutaneous endoscopic interlaminar lumbar discectomy versus open lumbar microdiscectomy at the L5-S1 level. PLoS One 2020;15:e0236296.

16 Wang $\mathrm{H}$, Zhou Y, Li C, et al. Risk factors for failure of single-level percutaneous endoscopic lumbar discectomy. J Neurosurg Spine 2015;23:320-5.

17 Choi K-C, Lee J-H, Kim J-S, et al. Unsuccessful percutaneous endoscopic lumbar discectomy: a single-center experience of 10,228 cases. Neurosurgery 2015;76:372-80. discussion 380-1; quiz 381.

18 Tenenbaum S, Arzi H, Herman A, et al. Percutaneous posterolatera Transforaminal endoscopic discectomy: clinical outcome complications, and learning curve evaluation. Surg Technol Int 2011;21:578-83.

19 Mayer HM. A history of endoscopic lumbar spine surgery: what have we learnt? Biomed Res Int 2019;2019:4583943.

20 Powell C. The Delphi technique: myths and realities. J Adv Nurs 2003;41:376-82.

21 Blaschke S-M, Lambert SD, Livingston PM, et al. Identifying priorities for cancer caregiver interventions: protocol for a three-round modified Delphi study. BMJ Open 2019;9:e024725.

22 Zamawe FC. The implication of using NVivo software in qualitative data analysis: evidence-based reflections. Malawi Med J 2015;27:13-15.

23 Merlin JS, Young SR, Azari S, et al. Management of problematic behaviours among individuals on long-term opioid therapy: protocol for a Delphi study. BMJ Open 2016;6:e011619.

24 Wu Y, Jin A, Xie G, et al. The 20 most important and most preventable health problems of China: a Delphi consultation of Chinese experts. Am J Public Health 2018;108:1592-8.

25 Jeste DV, Ardelt M, Blazer D, et al. Expert consensus on characteristics of wisdom: a Delphi method study. Gerontologist 2010;50:668-80.

26 Chang L-C, Chen Y-C, Wu FL, et al. Exploring health literacy competencies towards patient education programme for Chinesespeaking healthcare professionals: a Delphi study. BMJ Open 2017;7:e011772.

27 Cashin AG, McAuley JH, Lamb S. Items for consideration in a reporting guideline for mediation analyses: a Delphi study. BMJ Evid Based Med 2020.

28 Whiting $\mathrm{P}$, Wolff R, Mallett S, et al. A proposed framework for developing quality assessment tools. Syst Rev 2017;6:204.

29 Falzarano M, Pinto Zipp G. Seeking consensus through the use of the Delphi technique in health sciences research. J Allied Health 2013;42:99-105 